\title{
The Mus81 solution to resolution: generating meiotic crossovers without Holliday junctions
}

\author{
Nancy M. Hollingsworth ${ }^{1}$ and Steven J. Brill ${ }^{2,3}$ \\ ${ }^{1}$ Department of Biochemistry and Cell Biology, Institute for Cell and Developmental Biology, SUNY Stony Brook, Stony \\ Brook, New York 11794-5215, USA; ${ }^{2}$ Department of Molecular Biology and Biochemistry, Rutgers University, \\ Piscataway, New Jersey 08854, USA
}

During meiosis, recombination between homologous chromosomes creates a physical connection that is necessary for proper chromosome segregation at the first meiotic division. For many years, the paradigm used to explain the molecular mechanism of recombination has been the double-strand break repair (DSBR) model (Fig. 1; Szostak et al. 1983). (The definitions of the acronyms used in this review, in order of their appearance, can be found in Table 1.) One key feature of this model is that recombination is initiated by the formation of programmed DSBs (double-strand breaks). The meiosis-specific protein responsible for catalyzing these breaks is a topoisomerase-like protein called Spol1 (Bergerat et al. 1997; Keeney et al. 1997). The identification and functional analysis of Spo11 homologs from a wide variety of organisms, including worms, fruit flies, fission yeast, and mammals, indicates that repair of programmed DSBs is a universal feature of meiotic recombination (Keeney 2001). A second key feature of the model is the generation of a recombination intermediate called a double Holliday junction (dHJ). Differential resolution of this intermediate was proposed to determine the formation of crossover (CO) versus noncrossover (NCO) chromosomes. Recent advances indicate, however, that the decision of whether a recombination event will result in a $\mathrm{CO}$ or NCO chromosome occurs much earlier, soon after DSB formation (Allers and Lichten 2001a; Hunter and Kleckner 2001; Clyne et al. 2003). In addition, studies of a newly discovered endonuclease, Mus81, indicate that, in addition to $\mathrm{dHJ}$ resolution, COs may be formed by the processing of non-dHJ intermediates (Heyer et al. 2003; Osman et al. 2003). The decision of which crossover pathway to use appears to vary between organisms and may, therefore, be evolutionarily significant (de los Santos et al. 2003).

\footnotetext{
${ }^{3}$ Corresponding author.
}

E-MAIL brill@mbcl.rutgers.edu; FAX (732) 235-4880.

Article and publication are at http://www.genesdev.org/cgi/doi/10.1101/ gad.1165904.
The double-strand break repair model for meiotic recombination

A modified version of the Szostak et al. (1983) DSBR model is shown in Figure 1. Many of the recombination intermediates predicted by this model have been directly observed in budding yeast, and the gene products necessary for processing of different recombination intermediates have been identified (Smith and Nicolas 1998). In addition to SPO11, a large number of additional genes, whose roles are not yet understood, are required for DSB formation (Keeney 2001). The $5^{\prime}$-ends on either side of the break are resected to produce single-stranded (ss) 3'tails. Resection requires a trimeric complex of proteins containing Rad50, Xrs2, and Mre11 as well as Sae2/ Com 1 (Smith and Nicolas 1998). One of the 3'-tails invades the duplex of a homologous chromatid, thereby producing a D-loop or single end invasion (SEI) intermediate (Hunter and Kleckner 2001). Invasion of the homologous chromosome is promoted by two recA homologs, Rad51 and the meiosis-specific protein, Dmc1, as well as the presence of a barrier to sister-chromatid repair mediated by the meiosis-specific kinase Mek1 (Bishop et al. 1992; Wan et al. 2004). DNA synthesis extends the invading strand until the displaced strand in the D-loop overlaps sufficiently to anneal with the 3 '-tail on the other side of the break to create a second end capture (SEC) intermediate. DNA synthesis and ligation create an intact $\mathrm{dHJ}$-containing intermediate (Schwacha and Kleckner 1995). Resolution of both HJs in the same direction creates an NCO chromosome, whereas resolution of the junctions in opposite directions creates a CO chromosome. HJ resolvases from prokaryotes, such as RuvC and RusA, have been shown to make symmetrical nicks in strands of like polarity of HJs resulting in nicked duplex products (West 1997). Simple ligation of these nicked products restores intact duplexes. This version of the DSBR model assumes that eukaryotic $\mathrm{HJ}$ resolvases resolve HJs by a similar mechanism.

Recent data from the Lichten and Kleckner labs have challenged the idea that both COs and NCO chromosomes are derived from dHJs. For example, mutant con- 


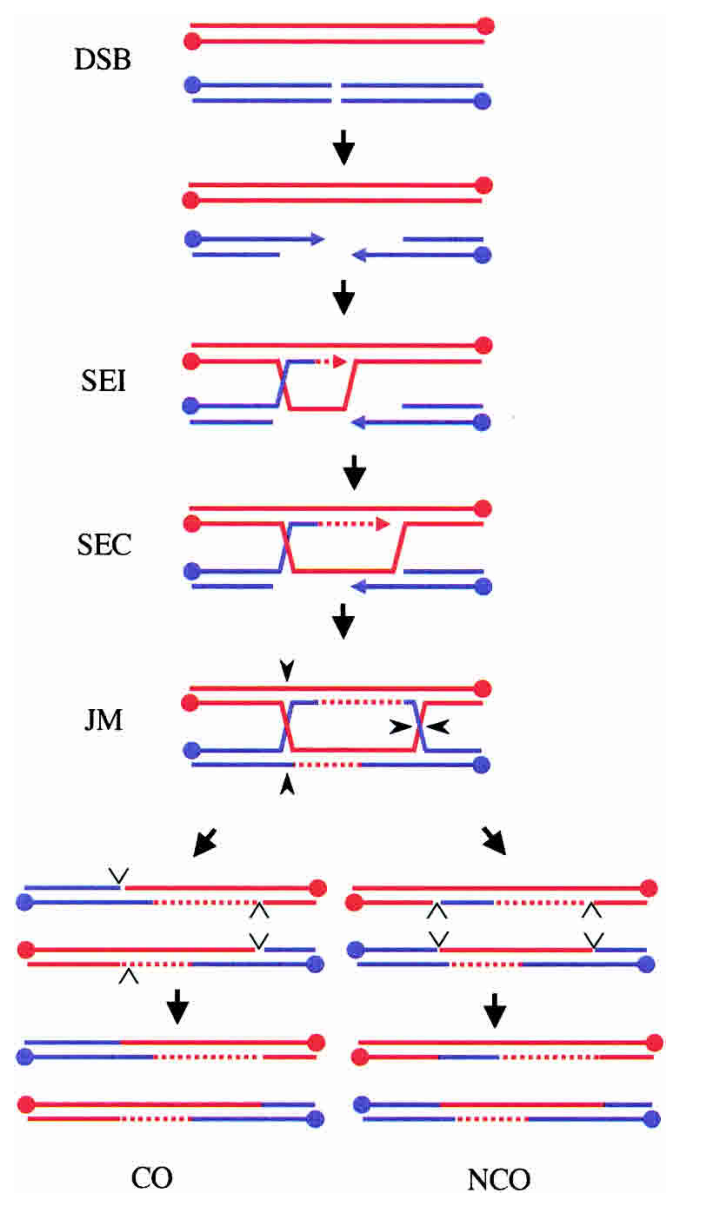

Figure 1. The DSBR model in meiosis. Shown are the duplex DNA strands of two non-sister chromatids from a pair of homologous chromosomes (Homolog 1 is red; Homolog 2 is blue). Meiotic DSBs are repaired following 5 '-end resection and the formation of an SEI intermediate. Elongation of the invading strand extends the D-loop until SEC initiates a second round of DNA synthesis. Ligation of the free ends creates a joint molecule containing dHJs. Resolution of the $\mathrm{dHJ}$ s in an opposite sense (e.g., black carets shown here) leads to the formation of a $\mathrm{CO}$, whereas cleavage in the same sense (e.g., cleavage of the crossed strands) results in an NCO. Resolution consists of two steps: symmetric cleavage to produce duplex DNA with nicks (open carets) and ligation of the nicks. $5^{\prime}$-Ends of the DNA strands are represented by dots, 3 '-ends are represented by arrows, and DNA synthesis is indicated by dashed lines.

ditions exist in which HJs are unresolved and COs are greatly reduced. Nevertheless, the formation of NCO chromosomes is unaffected (Allers and Lichten 2001a; Clyne et al. 2003). These results support the argument that repair of DSBs can occur by two separate pathways during budding yeast meiosis-a CO pathway that involves the formation of fully mature $\mathrm{dHJ}$ intermediates and an NCO pathway that does not (Fig. 2). NCOs are proposed to arise by displacement of the extended invading strand followed by reannealing to the $3^{\prime}$-tail on the other side of the break (synthesis-dependent strand annealing or SDSA; for review, see Paques and Haber 1999). Kinetic studies of the formation of different recombina- tion intermediates during meiosis in wild-type cells support this view (Allers and Lichten 2001a; Hunter and Kleckner 2001).

\section{The Mus81 ${ }^{\star}$ endonuclease functions in DNA metabolism in mitotic and meiotic cells}

Although many of the proteins necessary for mediating different steps of the DSBR pathway have been discovered, what has long remained elusive is the identification of a eukaryotic $\mathrm{HJ}$ resolvase. In the past few years, there has been much debate as to whether the Mus81 endonuclease may represent this Holy Grail of recombination (Haber and Heyer 2001; Heyer et al. 2003). The Mus81 protein shares homology with the XPF/Rad1 family of proteins involved in DNA nucleotide excision repair. Mus81 acts as a heterodimer with a second protein called Mms4 in the budding yeast, Saccharomyces cerevisiae, and Emel in the fission yeast, Schizosaccharomyces pombe (Boddy et al. 2001; Kaliraman et al. 2001). The human partner protein for Mus81 has been named both Mms4 and Emel (Ciccia et al. 2003; Ogrunc and Sancar 2003). For simplicity's sake, in this review Mus81/Mms4 and Mus81/Eme1 complexes will be indicated by Mus81*.

The MUS81/MMS4/EME1 genes were identified independently by several different labs, and mus $81 / \mathrm{mms} 4 /$ eme1 mutant phenotypes are consistent with a role in DNA repair (Haber and Heyer 2001). In mitotic cells, mus81/mms4 mutants exhibit sensitivity to DNA damaging agents such as methylmethane sulfonate (MMS) and camptothecin (CPT), which are believed to stall replication forks and generate DSBs as a result of problems with DNA replication (Prakash and Prakash 1977; Interthal and Heyer 2000; Doe et al. 2002; Bastin-Shanower et al. 2003). Mus $81^{\star}$ is not generally required for DSB repair in vegetative cells, however, as mus $81 / \mathrm{mms} 4$ mutants are resistant to DSB-producing agents such as ionizing radiation and bleomycin (Prakash and Prakash 1977; Xiao et al. 1998; Boddy et al. 2000; Interthal and Heyer 2000). Mus81 physically interacts with the budding yeast recombination protein Rad54 and with the fission

Table 1. Definition of acronyms in order of appearance

\begin{tabular}{ll}
\hline Acronym & \multicolumn{1}{c}{ Definition } \\
\hline DSBR & Double-strand break repair \\
DSB & Double-strand break \\
dHJ & Double Holliday junction \\
CO & Crossover \\
NCO & Noncrossover \\
SEI & Single end invasion \\
SEC & Second end capture \\
HJ & Holliday junction \\
SDSA & Synthesis-dependent strand annealing \\
MMS & Methylmethane sulfonate \\
CPT & Camptothecin \\
pRFs & Pseudoreplication forks \\
RLS & Regressed lagging strand \\
\hline
\end{tabular}




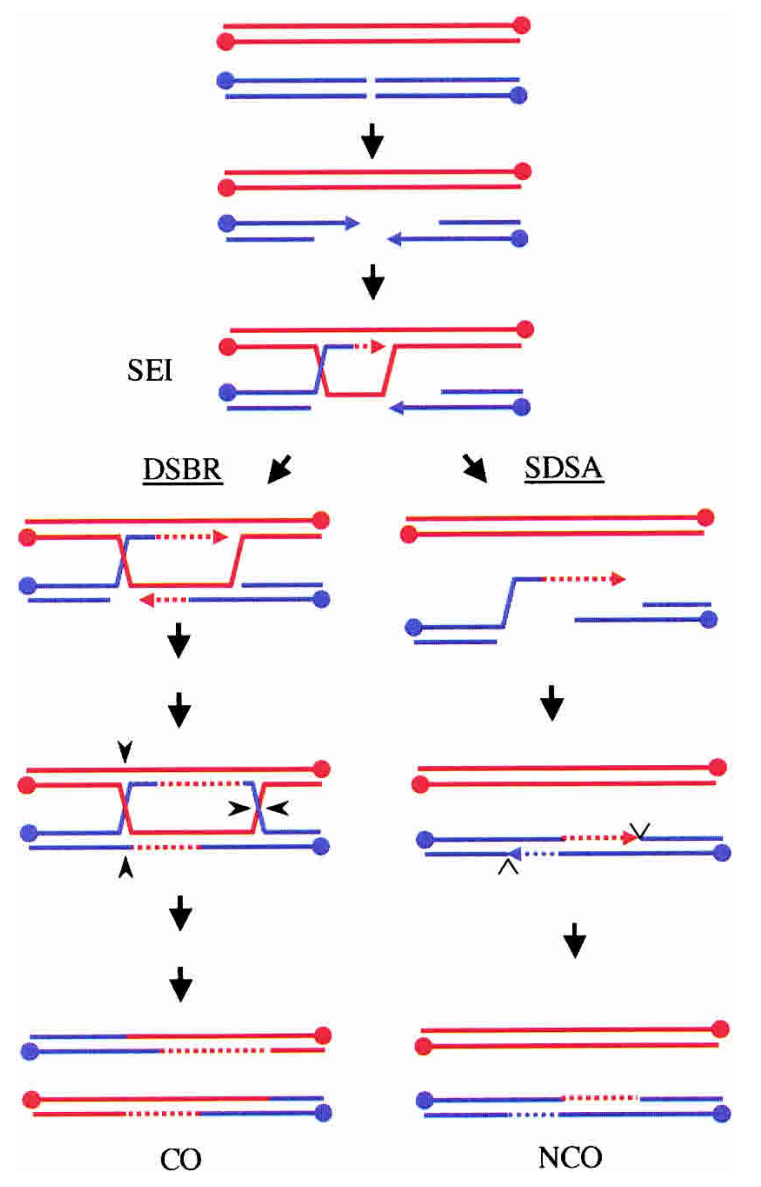

Figure 2. An alternative model for generating NCOs in budding yeast meiosis. Evidence at present suggests that SEI intermediates become committed to forming COs if they are repaired via the DSBR pathway, presumably through a biased resolution of $\mathrm{dHJ}$ s in the direction of COs (left pathway). The SEI intermediate may be disrupted, however, to allow repair to proceed via the SDSA pathway, resulting in NCO products (right pathway).

yeast DNA damage checkpoint kinase Cds1 (Boddy et al. 2000; Interthal and Heyer 2000). Mus81 is a nuclear protein and, in human cells, is recruited to sites of UVinduced damage specifically in $\mathrm{S}$ phase $(\mathrm{Fu}$ and Xiao 2003; Gao et al. 2003). These observations are consistent with the idea that Mus $81^{\star}$ plays a role in rescuing stalled replication forks. In addition, mus 81 and $m m s 4$ are synthetically lethal with mutants in SGS1 (RQH1 in fission yeast), a conserved $3^{\prime}-5^{\prime}$ DNA helicase known to be required for genome stability (Boddy et al. 2000; Mullen et al. 2001). This lethality can be rescued by preventing homologous recombination, suggesting that Mus $81^{*}$ acts on some type of recombination intermediate in mitotic cells (Fabre et al. 2002; Bastin-Shanower et al. 2003).

In both fission and budding yeast, mus $81 / \mathrm{mms} 4 / \mathrm{eme} 1$ mutants have severe meiotic phenotypes. In fission yeast, spore viability is reduced to $\leq 1 \%$, with high levels of chromosome missegregation (Boddy et al. 2001). In budding yeast, depending on temperature and strain background, up to $90 \%-100 \%$ of $m u s 81 / \mathrm{mms}^{4}$ diploids arrest in prophase as a result of unprocessed recombination intermediates triggering the meiotic recombination checkpoint (de los Santos et al. 2001, 2003). In those rare tetrads that are formed, spore viability is reduced to 40\% (Interthal and Heyer 2000; de los Santos et al. 2003). In both yeasts, these phenotypes are caused by a failure to repair meiosis-specific DSBs, as defects in meiotic progression and spore viability are rescued by mutations that prevent initiation of recombination (Boddy et al. 2001; de los Santos et al. 2001; Kaliraman et al. 2001). Clearly, Mus81 * plays an essential role in meiotic recombination in both $S$. pombe and $S$. cerevisiae. The critical question then has been, what is the mechanism of its action?

\section{Model I: Mus81 ${ }^{\star}$ cleaves intact $\mathrm{HJs}$}

Mus81 ${ }^{\star}$ was proposed to act at the $\mathrm{HJ}$ resolution step of the DSBR pathway, in part because partially purified Mus81* from fission yeast and human cells was observed to cleave intact HJs in vitro (Fig. 3D; Boddy et al. 2001; Chen et al. 2001). In addition, meiotic COs are reduced by mus 81 in both fission and budding yeast (although to different extents), whereas the frequency of NCOs is unaffected or even elevated (de los Santos et al. 2003; Osman et al. 2003; Smith et al. 2003). This is the expected result for a resolvase mutant assuming that $\mathrm{dHJ}$ are the precursors of COs and that NCOs form via a non-dHJ mechanism such as SDSA. It is important to note, however, that although there is direct support for this idea in budding yeast, there are no physical data demonstrating that $\mathrm{dHJ}$ are the precursors to COs in fission yeast. The most compelling evidence in support of the idea that Mus81 is an HJ resolvase is the fact that many of the mus $81 / \mathrm{mms} 4 / \mathrm{eme} 1$ mutant phenotypes, including meiotic chromosome missegregation and spore inviability in fission yeast, sensitivity to DNA damaging agents, and synthetic lethality with $r q h 1^{-}$are efficiently suppressed by overexpression of rusA, a bacterial resolvase with high specificity for HJs (Boddy et al. 2001; Doe et al. 2002; Odagiri et al. 2003). This argument assumes that rusA is directly substituting for the activity of Mus81*. However, the suppression could be indirect. For example, overexpression of rus $A$ also partially suppresses the defects exhibited by $r q h 1^{-}$cells that lack a 3 '-to-5' helicase, not an endonuclease (Doe et al. 2000).

The idea that Mus81* cleaves intact HJs was complicated by the discovery that recombinant budding yeast, fission yeast, and human Mus81* produced in Escherichia coli (designated rMus81 ${ }^{\star}$ ) have a significant preference for 3'-flap structures over intact HJs (Fig. 3, cf. A and D; Kaliraman et al. 2001; Doe et al. 2002; Ciccia et al. 2003). The primary determinant for optimal rMus81 * activity in vitro appears to be the presence of a nick in the substrate. Mapping studies indicate that the free $5^{\prime}$ end directs cleavage to the upstream strand one-half helical turn 5' of the nick (Bastin-Shanower et al. 2003; Osman et al. 2003). This rather nonspecific requirement results in efficient cleavage of several branched DNA substrates including 3'-flaps, pseudoreplication forks 


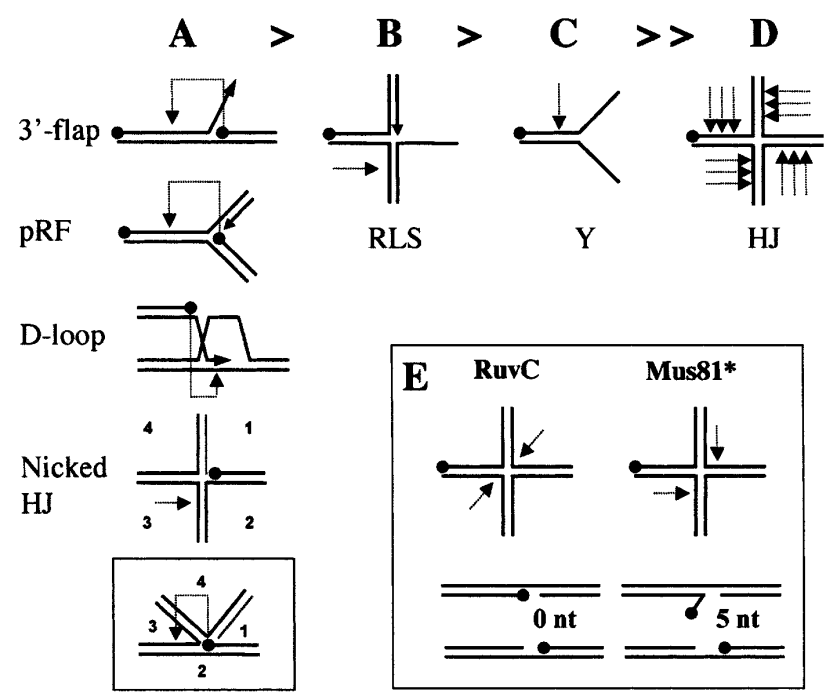

Figure 3. Substrate-specificity of Mus $81^{\star}$ in vitro. $(A-D)$ Summary of results from a variety of cleavage studies using rMus 81 * with substrates presented in order of cleavage efficiency. The most efficient substrates $(A)$ are cleaved well owing to the presence of a $5^{\prime}$-end at their branch junctions (indicated by a black dot). Cleavage of these substrates (light dotted arrow) is directed one-half helical turn upstream of this $5^{\prime}$-end (pRF, pseudoreplication fork). ( $A$, inset) This mechanism is consistent with the cleavage of nicked HJs in the strand opposing the nick. Efficient cleavage of the remaining substrates $(B-D)$ requires an excess of enzyme over substrate. A regressed lagging-strand substrate (RLS, panel $B$ ) is cleaved in the opposing strand, while a Y substrate $(C)$ is cleaved upstream of the branch junction. A further increase in enzyme concentration results in nicking of mobile HJs at multiple sites on the $5^{\prime}$-side of the mobile core. (E) Contrast of the HJ cleavage products obtained by symmetrical cleavage with RuvC (nicked duplexes) to the unligatable flap and gap products produced by Mus $81^{\star}$. These $5^{\prime}$-flap products may isomerize into $3^{\prime}$-flaps that are further processed by Mus $81^{\star}$ to produce only gapped molecules.

(pRFs), D-loops, and nicked HJs (Fig. 3A; Kaliraman et al. 2001; Doe et al. 2002; Gaillard et al. 2003; Osman et al. 2003; Whitby et al. 2003). When assayed in the presence of a molar excess of enzyme, however, a partial HJ substrate containing a single 3 '-end at the junction, sometimes referred to as a regressed lagging-strand substrate (RLS; Fig. 3B), is efficiently cleaved on the same strand as a nicked HJ (Gaillard et al. 2003; Osman et al. 2003). In addition, a simple $\mathrm{Y}$ substrate (Fig. 3C), which is preferred by the related XPF-ERCC1 nuclease, is cleaved upstream of the branch point. Although rMus81* endonuclease activity on the RLS and Y substrates is suboptimal, their cleavage suggests that there may be additional determinants for rMus81* activity in vitro. All groups agree that intact $\mathrm{HJs}$ are very poor substrates for rMus81* (Fig. 3D).

In contrast to rMus $81^{\star}$, partially purified preparations of epitope-tagged Mus81* from S. pombe cell extracts (TEV-Mus81 *) are active on intact HJs (Boddy et al. 2001; Gaillard et al. 2003). It has been suggested that the ability of TEV-Mus $81^{*}$ to cleave intact junctions is caused by the presence of a protein accessory factor or posttranslational modification (Gaillard et al. 2003). Mus81* partially purified from human cells, however, exhibits the same substrate specificity as the recombinant complexes, making it unlikely that the preference for 3 '-flaps and pRFs over HJs exhibited by the bacterially produced enzymes is caused by a lack of a posttranslational modification (Constantinou et al. 2002). Based on the preference of Mus81 * for nicked HJs, it has been proposed that Mus81* cleaves intact junctions by a "nick/counternick" mechanism (Gaillard et al. 2003). In this case, the first cut by Mus $81^{\star}$ is slow and rate-limiting. This nick makes the junction more flexible and a better substrate for Mus8 $81^{\star}$, and the second cut is proposed therefore to be nearly simultaneous with the first. An alternative explanation for the differences observed between TEVMus81* and rMus81, however, could derive from impurities present in the crude TEV-Mus $81^{\star}$ fraction. Resolving this paradox, therefore, will require comparison of rMus81/Eme1 from E. coli with highly purified Mus81* from $S$. pombe cells.

The HJ cleavage observed by Mus $81^{\star}$ is nonsymmetrical and therefore leaves flaps and gaps on the HJs that cannot be repaired simply by ligation (Fig. 3E; Boddy et al. 2001; Constantinou et al. 2002). Therefore, if Mus81 resolves intact $\mathrm{HJs}$, it does so by a non-RuvC/RusA-like mechanism. The idea that eukaryotic $\mathrm{HJ}$ resolution is mechanistically distinct from prokaryotic resolvases, while intriguing, is challenged by the discovery of a canonical RuvC-like activity in human cells. This activity, called Resolvase A, efficiently cuts intact HJs symmetrically to make ligatable, linear, nicked duplexes, is dependent on a branch migration activity, and is biochemically separable from Mus81* (Constantinou et al. 2002). Taken together, the biochemical data make it unlikely that the in vivo substrate of Mus81 is an intact HJ.

Further evidence that Mus $81^{\star}$ is not required to cleave intact HJs comes from meiotic studies using budding yeast (de los Santos et al. 2001, 2003). In this organism, dHJs can be directly analyzed (Schwacha and Kleckner 1995). Whereas HJs would be predicted to accumulate in a resolvase mutant, $m m s 4$ exhibited a decrease in the frequency of $\mathrm{dHJ}$ during meiosis compared with wild type. In addition, the effect of mms 4 on crossing over was, at most, twofold and appears to be dependent on chromosome size. Finally, overexpression of rus $A$ had no effect on the meiotic defects of either mus 81 or mms 4 in budding yeast (de los Santos et al. 2003). Therefore Mus81* is not required to generate the bulk of meiotic COs in budding yeast. This fact, coupled with the observation that $\mathrm{dHJ}$ s are the precursor to the majority of meiotic COs, provides compelling evidence that Mus $81^{\star}$ is not resolving intact $\mathrm{HJs}$ in budding yeast.

\section{Model II: Mus81 ^ cleaves 3 '-flaps generated by a partial strand displacement and annealing pathway of recombination}

mus81/mms4 diploids are clearly generating unprocessed recombination intermediates during budding yeast 
meiosis, as evidenced by the prophase arrest triggered by the meiotic recombination checkpoint and the fact that significant numbers of unrepaired DSBs remain at late times in meiosis. Based on the in vitro preference of Mus $81^{\star}$ for 3 '-flaps, as well as the discovery of a class of joint molecules in which heteroduplex DNA is to one side of the dHJs (Fig. 4; Allers and Lichten 2001b), an alternative model was proposed for Mus81 * function (de los Santos et al. 2001). In this model, a subset of dHJs was proposed to form by displacement of the 3 '-end from the extended D-loop and its subsequent annealing to the other side of the break. If the newly synthesized DNA on this strand is longer than the resected $3^{\prime}$-tail on the other side of the break, a $3^{\prime}$-flap could result that would then be cleaved by Mus81* (Fig. 4). Similar models invoking $3^{\prime}$-flap cleavage by Mus81* in mitotic cells have also been proposed (Fabre et al. 2002; Bastin-Shanower et al. 2003).

\section{Budding yeast has two genetically separable pathways for generating meiotic crossovers}

The 3 '-flap model (II) assumes that there are two pathways for generating COs, one that uses MUS81-MMS4 and one that does not. MSH4 and MSH5 encode meiosisspecific MutS homologs that have no role in mismatch repair, but instead are required to promote COs between homologous chromosomes (Ross-Macdonald and Roeder 1994; Hollingsworth et al. 1995). Similar to Mus81 and Mms4/Eme1, Msh4 and Msh5 function together as a heteroligomer (Pochart et al. 1997). Deletion of MSH4 and/ or MSH5 reduces crossing over two- to threefold and spore viability to $\sim 40 \%$. Interestingly, the COs that occur in msh4 mutants do not exhibit genetic interference

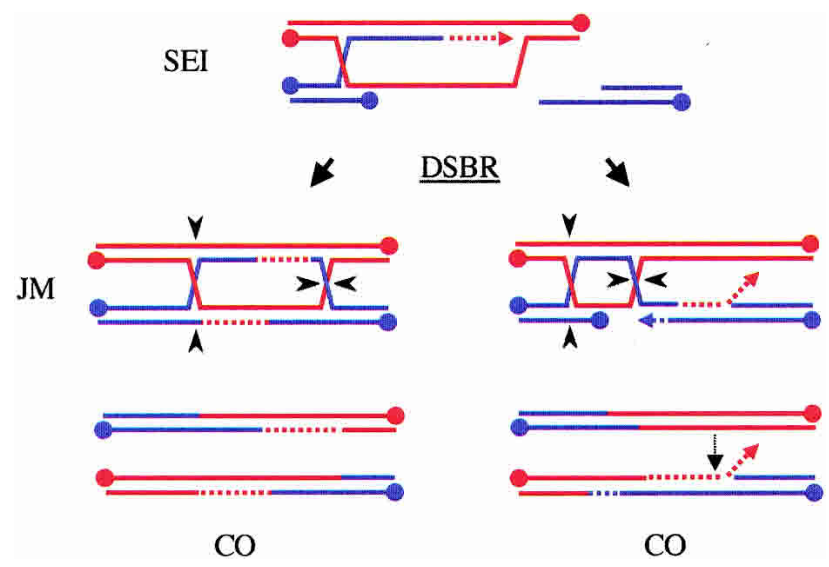

Figure 4. A role for Mus $81^{\star}$ in cleaving $3^{\prime}$-flaps. Model II proposes that most meiotic SEI intermediates are processed into COs via the DSBR pathway (left). In a subset of SEI intermediates, however, the invading strand may be elongated excessively (right). In this case, the second strand is captured, not by the D-loop, but by the elongated strand following displacement. Any 3 '-flaps that arise from strand annealing are substrates for Mus81* (light dotted arrow). In this pathway, heteroduplex DNA is found adjacent to the dHJs rather than between them.
(Novak et al. 2001). Interference is a phenomenon by which COs are distributed throughout the genome, perhaps to ensure that every chromosome receives at least one. Because COs are necessary for proper Meiosis I segregation, defects in interference may result in chromosome missegregation, or nondisjunction, at the first meiotic division. Consistent with a loss of interference, the spore lethality observed in $\mathrm{msh} 4 / \mathrm{msh} 5$ diploids is caused by chromosome nondisjunction (Ross-Macdonald and Roeder 1994; Hollingsworth et al. 1995). In contrast, the COs remaining in mms 4 diploids exhibit interference, and spore lethality is not caused by nondisjunction (de los Santos et al. 2003). msh5 mms4 diploids exhibit a sixfold decrease in crossing over and only $\sim 20 \%$ viable spores, confirming that these genes define two independent pathways for generating crossovers, an $\mathrm{MSH} 4$ MSH5-dependent interference pathway (designated Class I) and an MUS81-MMS4-dependent noninterference pathway (Class II).

The discovery of two different CO pathways in budding yeast may explain why spore viability is decreased to a much lesser extent in budding yeast mus 81 diploids compared with fission yeast mus 81 mutants $140 \%$ vs. $<1 \%$ ). In fission yeast, none of the COs display interference and the gene products required for the Class I pathway (e.g., MSH4 and MSH5) are absent from the $S$. pombe genome (Munz 1994; Villeneuve and Hillers 2001). Therefore, although the MUS81-MMS4 pathway accounts for a relatively minor fraction of COs in budding yeast, it is responsible for most, if not all, of the COs in fission yeast (Fig. 5).

Neither the HJ resolvase model nor the $3^{\prime}$-flap model for Mus81* function is satisfactory. As mentioned above, biochemical and genetic data do not support a role for Mus81* in the cleavage of intact HJs. A problem with the $3^{\prime}$-flap model is that it provides no way of easily discriminating between COs in the Class I and Class II pathways. Intact $\mathrm{dHJ}$ are postulated in the $3^{\prime}$-flap intermediates, and these must be resolved, presumably by the same resolvase that cleaves the junctions formed along the interference pathway (Fig. 3). Where does the MSH4MSH5 dependence come into play? The most disturbing feature about these two models is that they are organism-specific. That is, Mus $81^{\star}$ would have to behave like an $\mathrm{HJ}$ resolvase in fission yeast meiosis (Model I) and a 3 '-flap endonuclease in budding yeast meiosis (Model II). The most plausible model would be one that is consistent with both the in vitro preference of the enzyme and the genetic data from both yeasts. Recently, Whitby and colleagues have proposed a model for Mus81 function that satisfies these criteria (Osman et al. 2003).

\section{Model III: Mus81 ${ }^{\star}$ cleaves D-loops and half-junctions}

The most recent biochemical characterizations of rMus $81^{\star}$ and TEV-Mus $81^{\star}$ cleavage activity raise the possibility that D-loops and half-junctions are the in vivo substrates for the endonuclease, as opposed to $3^{\prime}$ flaps (Fig. 3; Gaillard et al. 2003; Osman et al. 2003). This idea is consistent with analyses that show that the pres- 
Figure 5. The role of Mus81* in creating COs without HJs. Model III proposes that meiotic SEI intermediates have three fates depending on the organism and pathway choice. All organisms use SDSA to produce NCOs (center). Class I interfering COs occur in organisms containing MSH4-MSH5 and arise from the DSBR pathway (left). Class II noninterfering COs are proposed to arise from the action of Mus81* on an SEC intermediate (right). The SEC intermediate provides targets for Mus $81^{\star}$ cleavage at the D-loop and at the half-junction (light dotted arrows). Cleavage at these sites results in an obligatory CO product containing $5^{\prime}$-flaps and gaps. Here the flaps are shown to isomerize into $3^{\prime}$-flaps prior to cleavage by a second round of Mus $81^{*}$ action. Fillingin of the gaps and ligation completes the $\mathrm{CO}$ product (not shown).

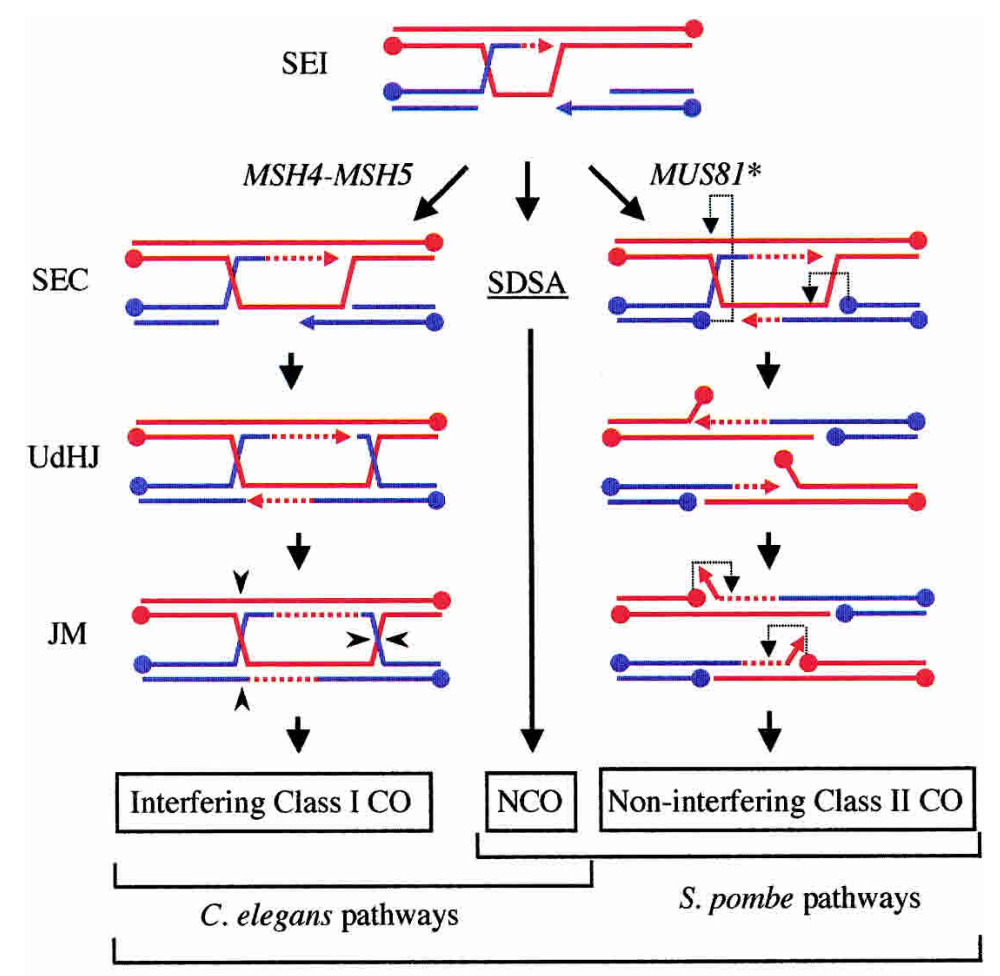

Mammalian (?) and S. cerevisiae pathways ence of a flap is not as important as the presence of a 5 '-nick (Bastin-Shanower et al. 2003). These findings led to the novel proposal that Mus81* generates COs without ever forming a dHJ intermediate (Fig. 5; Osman et al. 2003). In Model III, Mus81 cleaves a D-loop on one side of the break and the half-junction formed by SEC on the other side of the break (Fig. 5). This cleavage would result in gaps and/or $5^{\prime}$-flaps of $\sim 5 \mathrm{nt}$. Such flaps could isomerize to $3^{\prime}$-flaps and be cleaved by Mus $81^{*}$ or they could be processed by a $5^{\prime}$-flap endonuclease such as $\operatorname{Rad} 27$ in budding yeast or Rad2 in fission yeast (Alleva and Doetsch 1998; Kao et al. 2002). Interestingly, mus81 and $\mathrm{rad} 27$ are synthetically lethal in mitotic cells (Tong et al. 2001). Filling-in and ligation of the cleaved strands results in an obligatory $\mathrm{CO}$ without ever creating an intact $\mathrm{dHJ}$ intermediate (Osman et al. 2003). A variation of this model has been independently proposed by Heyer et al. (2003).

Model III beautifully reconciles the data between fission yeast and budding yeast. The assumption is that the SEI intermediate is the precursor to recombination in both organisms. In fission yeast, NCOs result from SDSA, whereas COs result almost exclusively from Mus81* cleavage (Fig. 5; Osman et al. 2003). In budding yeast, NCOs are also derived by SDSA, but COs can be generated by two independent pathways. The Class II, noninterfering COs result from Mus $81^{\star}$ cleavage, similar to fission yeast. Whether D-loop cleavage by Mus $81^{*}$ occurs prior to SEC as drawn in Osman et al. (2003) or after SEC (Fig. 5) is not yet known. For the Class I, interference-dependent COs, the SEC intermediate is ma- tured into a dHJ structure that is then resolved, presumably by an RuvC/RusA-like mechanism, to make COs. If Mus81 * cleaves D-loops before SEC, then MSH4-MSH5 could act at the step promoting formation of the SEC intermediate. Alternatively, if Mus $81^{\star}$ cleaves after SEC, MSH4-MSH5 might prevent this cleavage, thereby allowing maturation to dHJs. The MutS homolog, Msh2, is known to bind directly to HJs, so perhaps Msh4-Msh5 complexes bind directly to branched junctions as well (Alani et al. 1997; Marsischky et al. 1999). Msh4-Msh5 is part of the complex of proteins required for interference that includes Zip1, Zip2, and Zip3 (Agarwal and Roeder 2000). Binding of this complex to the junctions of developing recombination intermediates could create a physical barrier that prevents Mus81 * from cutting.

In budding yeast, Class II COs comprise a minority class that is derived independently from Class I COs and NCOs. Therefore, mus $81 / \mathrm{mms} 4$ mutants would be predicted to exhibit only a small decrease in COs and have no deleterious effects on gene conversion, which is exactly what is observed (de los Santos et al. 2003). In fission yeast, crossing over occurs primarily, if not entirely, by the Class II pathway. Therefore, by Model III, COs should be greatly reduced (Fig. 5). NCOs occur by SDSA after D-loop formation, and therefore should be unaffected. These predictions are consistent with the fission yeast recombination data (Osman et al. 2003; Smith et al. 2003). A strength of Model III, therefore, is that the differences observed in meiotic phenotypes between fission and budding yeast are explained, not by saying that Mus $81^{\star}$ cleaves different substrates in the two yeasts, 
but rather that cleavage of the same substrate has a different relative contribution to the total number of meiotic COs in each yeast.

The rusA suppression of fission yeast mus81 meiotic phenotypes could be explained if SEC intermediates can spontaneously proceed to $\mathrm{dHJ}$, thereby creating excellent substrates for RusA. If this occurs, then fission yeast has no way of normally resolving these $\mathrm{dHJs}$, given the very low spore viability of mus 81 mutants. The fact that rus $A$ overexpression is unable to suppress budding yeast mus81 meiotic defects may mean that formation of $\mathrm{dHJ}$ cannot proceed spontaneously in S. cerevisiae, but requires the activity of proteins such as Msh4-Msh5. Alternatively, the failure of rusA to suppress mus81 budding yeast meiotic phenotypes could be due to the very significant differences in meiotic chromosome behavior exhibited by the two yeasts. During budding yeast meiosis, homologous chromosomes become physically associated by the formation of a meiosis-specific proteinaceous structure called the synaptonemal complex (SC; Roeder 1997). In contrast, in fission yeast, homologs do not synapse and no SC is formed (Bahler et al. 1993). The SC may, therefore, prevent RusA from accessing recombination intermediates during budding yeast meiosis. Consistent with this idea, rus $A$ is able to suppress some mus $81 / \mathrm{mms} 4$ phenotypes in vegetatively growing budding yeast cells where the SC is absent (Bastin-Shanower et al. 2003; Odagiri et al. 2003).

\section{The semantics of resolution}

Much of the controversy surrounding Mus81 * has been the issue of whether it is an $\mathrm{HJ}$ resolvase or not. Both genetic and biochemical data support the argument against intact $\mathrm{HJs}$ being the target of Mus81*. Model III suggests that Mus81 cleavage of non-HJ intermediates results in the formation of COs after further DNA processing events. If this model is correct, Mus81 could be considered to "resolve" recombination intermediates. For many people, however, the term "resolvase" conjures up a specific paradigm-that of RuvC/RusA-like nucleases cleaving strands of like polarity in $\mathrm{HJ}$ s to create ligatable, nicked, linear duplexes. For clarity, therefore, it might be most accurate to refer to Mus $81{ }^{\star}$, not as a resolvase, but as a "nicked junction endonuclease."

\section{Conclusion}

For years, recombination models have assumed that COs in eukaryotic cells would follow the paradigm from bacteria of resolution of intact HJs by an RuvC/RusA-like resolvase. The discovery of Mus81* has raised the possibility that cells have evolved at least two ways for generating COs, the Class I pathway, which follows the paradigm of intact $\mathrm{HJ}$ resolution, and the Class II pathway, which may not. During evolution, the choice of which pathway(s) to use has varied with different organisms (Fig. 5). Fission yeast appears to depend exclusively on the Class II pathway, which may be because of the failure of homologous chromosomes to synapse. In contrast, the majority of COs in budding yeast occur by the Class I pathway, and the Class II pathway may exist primarily as a backup to ensure that any recombination intermediates that fail to be converted into intact $\mathrm{dHJ}$ be repaired. In worms, deletion of $\mathrm{MSH} 4$ or MSH5 eliminates all COs, suggesting that meiotic COs in this organism rely exclusively on the Class I pathway (Fig. 5; Zalevsky et al. 1999; Kelly et al. 2000).

One outcome in having two independent ways of generating COs is the ability to modulate interference. Interestingly, mms 4 exhibits a stronger phenotype on a small chromosome that displays a lower level of interference than on a large chromosome on which interference is stronger (Kaback et al. 1999; de los Santos et al. 2003). Therefore, the degree of interference may be regulated by altering the frequency with which the different CO pathways are used. The two extreme cases are fission yeast, where there is no interference and COs are generated primarily, if not exclusively, by the Class II pathway, and worms, where interference is strong (Hillers and Villeneuve 2003) and the Class I pathway apparently generates all COs. It is possible that mammals, like budding yeast, use both CO pathways during meiosis. Mammalian chromosomes exhibit interference, and $\mathrm{MSH} 4$ and MSH5 are meiosis-specific genes required for the proper execution of meiosis; Mus $81^{\star}$ activity has been detected in somatic cells (de Vries et al. 1999; Broman and Weber 2000; Kneitz et al. 2000). However, whether mammals follow the worm paradigm, in which all COs are $M S H 4 / M S H 5$-dependent, or the budding yeast paradigm, in which a minority of COs are generated by Mus $81^{\star}$, is as yet unknown. The study of this very interesting enzyme has therefore suggested a novel mechanism for generating meiotic COs and may ultimately shed light on how the distribution of COs is regulated in different organisms.

\section{Acknowledgments}

We thank Neil Hunter and Michael Lichten for helpful comments on the manuscript. We are grateful to W. Heyer, G. Smith, C. McGowan, P. Russell, and M. Whitby for communicating ideas/results prior to publication. Research in N.M.H.'s laboratory was supported by NIH grant GM50717 and March of Dimes grant 1-FY02-754, and research in S.J.B.'s laboratory is supported by NIH grant GM67956.

\section{References}

Agarwal, S. and Roeder, G.S. 2000. Zip3 provides a link between recombination enzymes and synaptonemal complex proteins. Cell 102: 245-255.

Alani, E., Lee, S., Kane, M.F., Griffith, J., and Kolodner, R.D. 1997. Saccharomyces cerevisiae MSH2, a mispaired base recognition protein, also recognizes Holliday junctions in DNA. J. Mol. Biol. 265: 289-301.

Allers, T. and Lichten, M. 2001a. Differential timing and control of noncrossover and crossover recombination during meiosis. Cell 106: 47-57. 
- 2001b. Intermediates of yeast meiotic recombination contain heteroduplex DNA. Mol. Cell 8: 225-231.

Alleva, J.L. and Doetsch, P.W. 1998. Characterization of Schizosaccharomyces pombe $\operatorname{Rad} 2$ protein, a FEN-1 homolog. Nucleic Acids Res. 26: 3645-3650.

Bahler, J., Wyler, T., Loidl, J., and Kohli, J. 1993. Unusual nuclear structures in meiotic prophase of fission yeast: A cytological analysis. J. Cell Biol. 121: 241-256.

Bastin-Shanower, S.A., Fricke, W.M., Mullen, J.R., and Brill, S.J. 2003. The mechanism of Mus81-Mms4 cleavage site selection distinguishes it from the homologous endonuclease Rad1-Rad10. Mol. Cell. Biol. 23: 3487-3496.

Bergerat, A., de Massy, B., and Gadelle, D. 1997. An atypical topoisomerase II from Archaea with implications for meiotic recombination. Nature 386: 414-417.

Bishop, D.K., Park, D., Xu, L., and Kleckner, N. 1992. DMC1: A meiosis-specific yeast homolog of $E$. coli recA required for recombination, synaptonemal complex formation and cell cycle progression. Cell 69: 439-456.

Boddy, M.N., Lopez-Girona, A., Shanahan, P., Interthal, H., Heyer, W.D., and Russell, P. 2000. Damage tolerance protein mus81 associates with the FHA1 domain of checkpoint kinase cds1 [In Process Citation]. Mol. Cell. Biol. 20: 87588766.

Boddy, M.N., Gaillard, P.-H.L., McDonald, W.H., Shanahan, P., Yates III, J.R., and Russell, P. 2001. Mus81-Eme1 are essential components of a Holliday junction resolvase. Cell 107: 537-548.

Broman, K.W. and Weber, J.L. 2000. Characterization of human crossover interference. Am. J. Hum. Genet. 66: 1911-1926.

Chen, X.-B., Melchionna, R., Denis, C.-M., Gaillard, P.-H.L., Blasina, A., Van de Weyer, I., Boddy, M.N., Russell, P., Vialard, J., and McGowan, C.H. 2001. Human Mus81-associated endonuclease cleaves Holliday junctions in vitro. Mol. Cell 8: $1117-1127$.

Ciccia, A., Constantinou, A., and West, S.C. 2003. Identification and purification of the human Mus81/Eme1 endonuclease. J. Biol. Chem. 278: 25172-25178.

Clyne, R.K., Katis, V.L., Jessop, L., Benjamin, K.R., Herskowitz, I., Lichten, M., and Nasmyth, K. 2003. Polo-like kinase Cdc5 promotes chiasmata formation and cosegregation of sister centromeres at meiosis I. Nat. Cell Biol. 5: 480-485.

Constantinou, A., Chen, X.B., McGowan, C.H., and West, S.C. 2002. Holliday junction resolution in human cells: Two junction endonucleases with distinct substrate specificities. EMBO J. 21: 5577-5585.

de los Santos, T., Loidl, J., Larkin, B., and Hollingsworth, N.M. 2001. A role for MMS4 in the processing of recombination intermediates during meiosis in Saccharomyces cerevisiae. Genetics 159: 1511-1525.

de los Santos, T., Hunter, N., Lee, C., Larkin, B., Loidl, J., and Hollingsworth, N.M. 2003. The Mus81/Mms4 endonuclease acts independently of double-Holliday junction resolution to promote a distinct subset of crossovers during meiosis in budding yeast. Genetics 164: 81-94.

de Vries, S.S., Baart, E.B., Dekker, M., Siezen, A., de Rooij, D.G., de Boer, P., and te Riele, H. 1999. Mouse MutS-like protein Msh5 is required for proper chromosome synapsis in male and female meiosis. Genes \& Dev. 13: 523-531.

Doe, C.L., Dixon, J., Osman, F., and Whitby, M.C. 2000. Partial suppression of the fission yeast $\mathrm{rqh} 1^{-}$phenotype by expression of a bacterial Holliday junction resolvase. EMBO $J$. 19: $2751-2762$.

Doe, C.L., Ahn, J.S., Dixon, J., and Whitby, M.C. 2002. Mus81Emel and Rqh1 involvement in processing stalled and collapsed replication forks. J. Biol. Chem. 277: 32753-32759.
Fabre, F., Chan, A., Heyer, W.D., and Gangloff, S. 2002. Alternate pathways involving Sgs1/Top3, Mus81/ Mms4, and Srs2 prevent formation of toxic recombination intermediates from single-stranded gaps created by DNA replication. Proc. Natl. Acad. Sci. 99: 16887-16892.

$\mathrm{Fu}, \mathrm{Y}$. and Xiao, W. 2003. Functional domains required for the Saccharomyces cerevisiae Mus81-Mms4 endonuclease complex formation and nuclear localization. DNA Repair 2: $1435-1447$.

Gaillard, P.-H.L., Noguchi, E., Shanahan, P., and Russell, P. 2003. The endogenous Mus81-Emel complex resolves Holliday junctions by a nick and counternick mechanism. Mol. Cell 12: 747-759.

Gao, H., Chen, X.-B., and McGowan, C.H. 2003. Mus81 endonuclease localizes to nucleoli and to regions of DNA damage in human S-phase cells. Mol. Biol. Cell 14: 4826-4834.

Haber, J.E. and Heyer, W.D. 2001. The fuss about Mus81. Cell 107: 551-554.

Heyer, W.D., Ehmsen, K.T., and Solinger, J.A. 2003. Holliday junctions in the eukaryotic nucleus: Resolution in sight? Trends Biochem. Sci. 28: 548-557.

Hillers, K.J. and Villeneuve, A.M. 2003. Chromosome-wide control of meiotic crossing over in C. elegans. Curr. Biol. 13: $1641-1647$.

Hollingsworth, N.M., Ponte, L., and Halsey, C. 1995. MSH5, a novel MutS homolog, facilitates meiotic reciprocal recombination between homologs in Saccharomyces cerevisiae but not mismatch repair. Genes \& Dev. 9: 1728-1739.

Hunter, N. and Kleckner, N. 2001. The single-end invasion: An asymmetric intermediate at the double-strand break to double-Holliday junction transition of meiotic recombination. Cell 106: 59-70.

Interthal, H. and Heyer, W.D. 2000. MUS81 encodes a novel helix-hairpin-helix protein involved in the response to UVand methylation-induced DNA damage in Saccharomyces cerevisiae. Mol. Gen. Genet. 263: 812-827.

Kaback, D.B., Barber, D., Mahon, J., Lamb, J., and You, J. 1999. Chromosome size-dependent control of meiotic reciprocal recombination in Saccharomyces cerevisiae: The role of crossover interference. Genetics 152: 1475-1486.

Kaliraman, V., Mullen, J.R., Fricke, W.M., Bastin-Shanower, S.A., and Brill, S.J. 2001. Functional overlap between the Sgs1-Top3 and the fork endonuclease Mms4-Mus81: Potential mechanisms for re-establishing replication forks. Genes \& Dev. 15: 2730-2740.

Kao, H.I., Henricksen, L.A., Liu, Y., and Bambara, R.A. 2002. Cleavage specificity of Saccharomyces cerevisiae flap endonuclease 1 suggests a double-flap structure as the cellular substrate. J. Biol. Chem. 277: 14379-14389.

Keeney, S. 2001. Mechanism and control of meiotic recombination initiation. Curr. Top. Dev. Biol. 52: 1-53.

Keeney, S., Giroux, C.N., and Kleckner, N. 1997. Meiosis-specific DNA double strand breaks are catalyzed by Spo11, a member of a widely conserved protein family. Cell 88: 375 384.

Kelly, K.O., Dernberg, A.F., Stanfield, G.M., and Villeneuve, A.M. 2000. Caenorhabditis elegans msh-5 is required for both normal and radiation-induced meiotic crossing over but not for completion of meiosis. Genetics 156: 617-630.

Kneitz, B., Cohen, P.E., Avdievich, E., Zhu, L., Kane, M.F., Hou Jr., H., Kolodner, R.D., Kucherlapati, R., Pollard, J.W., and Edelmann, W. 2000. MutS homolog 4 localization to meiotic chromosomes is required for chromosome pairing during meiosis in male and female mice. Genes \& Dev. 14: 10851097.

Marsischky, G.T., Lee, S., Griffith, J., and Kolodner, R.D. 1999. 
Saccharomyces cerevisiae MSH2/6 complex interacts with Holliday junctions and facilitates their cleavage by phage resolution enzymes. J. Biol. Chem. 274: 7200-7206.

Mullen, J.R., Kaliraman, V., Ibrahim, S.S., and Brill, S.J. 2001. Requirement for three novel protein complexes in the absence of the Sgs1 DNA helicase in Saccharomyces cerevisiae. Genetics 157: 103-118.

Munz, P. 1994. An analysis of interference in the fission yeast Schizosaccharomyces pombe. Genetics 137: 701-707.

Novak, J.E., Ross-Macdonald, P.B., and Roeder, G.S. 2001. The budding yeast Msh4 protein functions in chromosome synapsis and the regulation of crossover distribution. Genetics 158: $1013-1025$.

Odagiri, N., Seki, M., Onoda, F., Yoshimura, A., Watanabe, S., and Enomoto, T. 2003. Budding yeast mms 4 is epistatic with rad52 and the function of Mms 4 can be replaced by a bacterial Holliday junction resolvase. DNA Repair (Amst) 2: 347358.

Ogrunc, M. and Sancar, A. 2003. Identification and characterization of human MUS81-MMS4 structure-specific endonuclease. J. Biol. Chem. 278: 21715-21720.

Osman, F., Dixon, J., Doe, C.L., and Whitby, M.C. 2003. Generating crossovers by resolution of nicked Holliday junctions: A role for Mus81-Eme1 in meiosis. Mol. Cell 12: 761-774.

Paques, F. and Haber, J.E. 1999. Multiple pathways of recombination induced by double-strand breaks in Saccharomyces cerevisiae. Microbol. Mol. Biol. Rev. 63: 349-404.

Pochart, P., Woltering, D., and Hollingsworth, N.M. 1997. Conserved properties between functionally distinct MutS homologs in yeast. J. Biol. Chem. 48: 30345-30349.

Prakash, L. and Prakash, S. 1977. Isolation and characterization of MMS-sensitive mutants of Saccharomyces cerevisiae. Genetics 86: 33-55.

Roeder, G.S. 1997. Meiotic chromosomes: It takes two to tango. Genes \& Dev. 11: 2600-2621.

Ross-Macdonald, P. and Roeder, G.S. 1994. Mutation of a meiosis-specific MutS homolog decreases crossing over but not mismatch correction. Cell 79: 1069-1080.

Schwacha, A. and Kleckner, N. 1995. Identification of double Holliday junctions as intermediates in meiotic recombination. Cell 83: 783-791.

Smith, K.N. and Nicolas, A. 1998. Recombination at work for meiosis. Curr. Opin. Genet. Dev. 8: 200-211.

Smith, G.R., Boddy, M.N., and Russell, P. 2003. Fission yeast Mus81-Eme1 Holliday junction resolvase is required for meiotic crossing-over but not for gene conversion. Genetics (in press).

Szostak, J.W., Orr-Weaver, T.L., Rothstein, R.J., and Stahl, F.W. 1983. The double strand-break model for recombination. Cell 33: 25-35.

Tong, A.H., Evangelista, M., Parsons, A.B., Xu, H., Bader, G.D., Page, N., Robinson, M., Raghibizadeh, S., Hogue, C.W., Bussey, H., et al. 2001. Systematic genetic analysis with ordered arrays of yeast deletion mutants. Science 294: 2364-2368.

Villeneuve, A.M. and Hillers, K.J. 2001. Whence meiosis? Cell 106: 647-650.

Wan, L., de los Santos, T., Zhang, C., Shokat, K., and Hollingsworth, N.M. 2004. Mek1 kinase activity functions downstream of RED1 in the regulation of meiotic DSB repair in budding yeast. Mol. Biol. Cell 15: 11-23.

West, S.C. 1997. Processing of recombination intermediates by the RuvABC proteins. Annu. Rev. Genet. 31: 213-244.

Whitby, M.C., Osman, F., and Dixon, J. 2003. Cleavage of model replication forks by fission yeast Mus81-Emel and budding yeast Mus81-Mms4. J. Biol. Chem. 278: 6928-6935.

Xiao, W., Chow, B.L., and Milo, C.N. 1998. Mms4, a putative transcriptional (co)activator, protects Saccharomyces cerevisiae cells from endogenous and environmental DNA damage. Mol. Gen. Genet. 257: 614-623.

Zalevsky, J., MacQueen, A.J., Duffy, J.B., Kemphues, K.J., and Villeneuve, A.M. 1999. Crossing over during Caenorhabditis elegans meiosis requires a conserved MutS-based pathway that is partially dispensable in budding yeast. Genetics 153: $1271-1283$. 


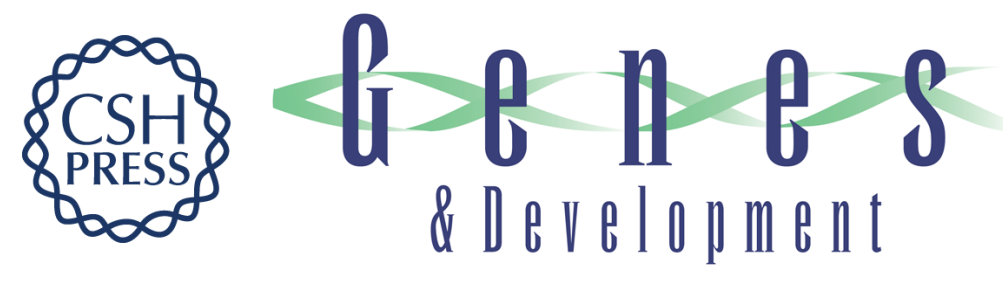

\section{The Mus81 solution to resolution: generating meiotic crossovers without Holliday junctions}

Nancy M. Hollingsworth and Steven J. Brill

Genes Dev. 2004, 18:

Access the most recent version at doi:10.1101/gad.1165904

References This article cites 58 articles, 30 of which can be accessed free at: http://genesdev.cshlp.org/content/18/2/117.full.htmI\#ref-list-1

License

Email Alerting

Receive free email alerts when new articles cite this article - sign up in the box at the top Service right corner of the article or click here.

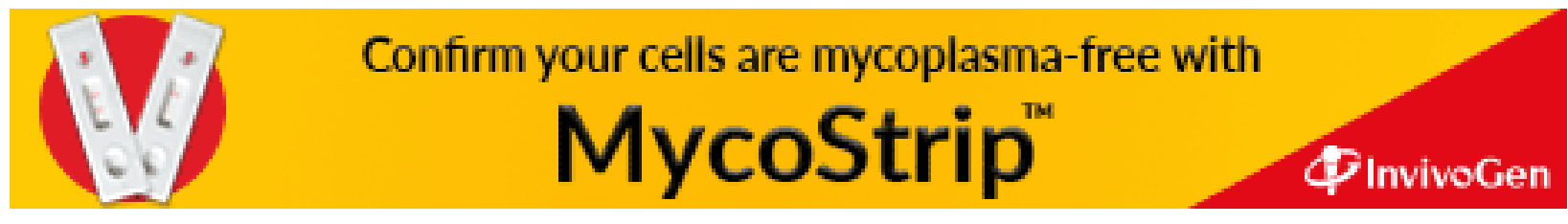

